\title{
Algunas consideraciones sobre las aplicaciones de forceps
}

\author{
Doctor Miguel Páez V., Residente de la Clínica David Retrepo, \\ de Bogotá.
}

No cabe duda de que la introducción del forceps a la práctica obstétrica, constituyó uno de los mayores progresos en esta rama de la ciencia, pero como sucede siempre con lo que no es bien conocido ni está completamente estudiado, se abuso de su em. pleo, con lo cual el saldo de sus aplicaciones no era favorable en el grado en que lo es hoy, gracias a la experiencia que de él se tiene $y$ de los estudios llevados a cabo, que hacen del forceps un instrumento casi inocuo, necesario y a veces indispensable, con normas y leyes precisas que han hecho que se imponga en la práctica obstétrica como un elemento verđaderamente insustituible:

El ideal médico, tratándose de partos, sería que éstos se realizaran en forma fisiológica y natural, y que el obstetra no tuviera que intervenir sino ayudando a la naturalezia en su obra creadora. Pero sucede muy a menudo que la fisiolozía se trastorna, y lo que debiera ser natural se hace aberrante, colocando al médico en condiciones difíciles y a veces desesperantes.

Es en algunos de estos disturbios en los que el forceps encuentra su indicación precisa, resolviendo así una situación alarmante. Pero esto no quiere decir en ninguna form que para todos los partos difíciles la solución inmediata sea una aplicación de forceps, lo cual nos llevaría a la resultante lógica, que sería un intervencionismo desmedido, un abuso sin fundamento del forceps, que aunque en algunos casos es una feliz solución, en otros sería un triste fracaso. El forceps es un elemento valioso para el obstetra, pero también le está vedado invadir terrenos que no le per temecen: Para su aplicación existen normas y leyes precisas que el médico debe tener muy presentes antes de intervenir. 
¿Cuáles son estas condiciones? Como primera medida, que la aplicación del forceps esté indicada; segundo, que el cuello del útero esté completamente dilatado, o que sea posible completar la dilatación manualmente; tercero, que la bolsa de las aguas esté rota; cuarto, que la presentación esté encajada y que la pelvis sea capaz. Las primeras indicaciones o condiciones son logicas y no admiten reparo alguno. En cuanto al encajamiento, la conducta varía de acuerdo con el criterio del obstetra. En este sentido existen dos escuelas: la que proscribe rotundamente las aplicaciones de forceps sobre cabeza no encajada, y a la cual pertenecen obstetras muy eminentes, quienes aducen que la constitución del forceps y de la pelvis contraindican las tomas por encima del estrecho superior, por inconvenientes y perjudiciales, y la escuela de los que defienden y hacen aplicaciones altas de forceps, aduciendo el argumento de que son posibles y se han hecho con buenos resultados, en condiciones que son favorables para estas aplicaciones, y con la condición de que el que las hago tenga gran pericia y habilidad, porque de lo contrario las consecuencias son desastrosas. Nosotros no entramos aquí a considerar la razón o sinrazón de estas dos tendencias, respetando el criterio y la manera de obrar de cada cual. Solamente queremos anotar que hemos visto hacer aplicacioens altas de forceps con buenos resultados.

Los comentarios a las aplicaciones de forceps que originaron el presente trabajo se basan en observaciones de historias clínicas de los partos atendidos en la Clínica de Maternidad David Restrepo, en los cuales hemos tenido intervención directa o indirecta, haciendo las aplicaciones o colaborando con el obstetra que lo haya hecho, en el curso de quince meses. Se trata, pues, de una estadística no particular, pero sí relativamente perteneciente a nosotros.

Durante el tiempo mencionado han sido atendidus en la Clinica Restrepo, setecientos ochenta y nueve (789) partos, entre los cuales quedan incluídos los partos prematuros, los partos a término, los partos espontáneos, las partos con forceps y las cesáreas realizadas. Sobre este total se han hecho sesenta y una (61) aplicaciones de forceps, lo que reducido a porcentaje nos da una cifra de $7.73 \%$. Estas aplicaciones en relación con la altura a la cual fueron colocados, se distribuyen así:

Estrecho Superior: 3. Estrecho Medio: 24. Estrecho Inferior: 34. 
Por razón de la causa que obligó a intervenir para terminar el parto artificialmente, se dividen así:

Cardiopatías, 3; sufrimiento fetal, 25; profilácticos, 1; procidencia del cordón, 2; demora en rotación y expulsión, 27; distocia de encajamiento, 3 .

En general, en el total de los sesenta y un casos se presentaron circulares del cordón, en veinte.

Frecuencia, según las variedades de posición:

O. D. P., 32; O. I. A., 17; O. I. T., 7; O. I. P., 4; N. I. T., 1.

Lo cual da para variedades posteriores un total de 36 casos; para variedades anteriores, 17, y para variedades transversas, 8.

En relación con la paridad, los casos se reparten asi:

Primíparas, 41; multiparas, 20.

Incluyendo entre las multiparas tres casos, cuyos primeros embarazos terminaron por aborto en los primeros meses, y dos secundiparas que tuvieron cesárea en el primer parto.

De acuerdo con los diámetros pélvicos, tomando como base el conjugado externo o de Baudelocque, los casos se reparten asi:

De 20 centímetros o más, 31; entre 19 y 20, 20; entre 18 y 19 , 7, y entre 17 y 18,3 .

Se tomaron radio pelvimetrías en nueve casos, todos los cua. les dieron datos de diámetros pequeños.

Partos inducidos, 3; iniciación espontánea del trabajo, 58; embarazos concomitantes con hidramnios, 4; embarazos que evolucionaron con toxemia, 4; ruptura prematura de las membranas, 8 .

En relación con las lesiones maternas consecutivas a estas aplicaciones de forceps, se tuvo el siguiente resultado:

Episiotomís, 35; desgarros, 8; sin episiotomía n1 desgarro, 18.

Resultados fetales: fetos a término, 57; prematuros, 4; con respiración espontánea, 55; respiración retardada, 5; no respiraron, 1.

Fetos vivos, 60; muertos, 1; muertes neo natales, 3.

Frecuencia, según la edad de la paciente:

\section{Primíparas}

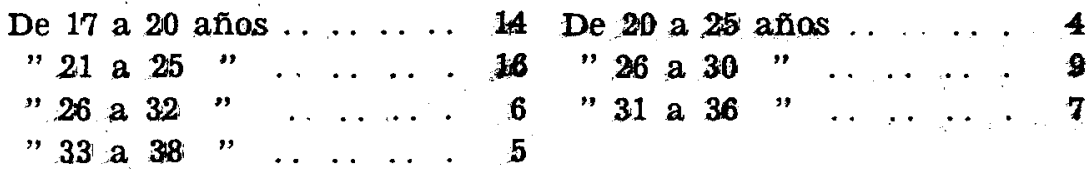


Los antecedentes obstétricos de las multíparas iueron así:

Partos normales, 11; partos con forceps, 3; con cesárea, 2; con abortos en sus primeros embarazos, 3 ; con partos prematuros, 1.

Según la duración del trabajo se reparten asi:

\begin{tabular}{|c|c|c|c|c|c|c|c|c|c|c|c|}
\hline De & 1 a 5 & horas & $\cdots$ & . & 4 & De & 25 & a 30 & horas & $\cdots$ & \\
\hline ", & 5 a 10 & ", & & $\therefore$ & 19 & ", & 30 & a 35 & $"$ & . & \\
\hline & 10 a 15 & " & 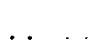 & & 12 & $"$ & 35 & a 37 & $"$ & & \\
\hline & 15 a 20 & $"$ & $\ldots$ & . & 11 & $"$ & 55 & a 56 & $"$ & $\ldots \ldots$ & \\
\hline & 20 a 25 & $"$ & $\ldots \ldots$ & & 4 & $"$ & 69 & $a-$ & " & $\ldots \ldots$ & \\
\hline
\end{tabular}

\section{Comentarios a los resultados anteriores.}

Como puede notarse, entre setecientos ochenta $y$ nueve partos (789) atendidos, se han aplicado sesenta y un forceps (61), o lo que es 10 mismo un siete con setenta y tres $(7.73 \%)$ por ciento. No creemos que resulte muy alto este porcentaje, si tenemos en cuenta que en el New York Lying In Hospital, las intervenciones de forceps indicados ascienden a un diez (10\%) por ciento, sobre el total de los partos atendidos.

Ahora bien: si tomamos en cuenta la altura a que fueron hechas las aplicaciones, encontramos que el mayor nimero corresponde a aplicaciones sobre el estrecho inferior, en proporción del $\mathbf{5 5 . 7 \%}$, siguiendo las aplicaciones sobre el estrecho medio, con un $39.3 \%$ y en el estrecho superior con un 5\%, lo cual está de acuerdo con lo que se ve en la práctica diaria, en que la mayoría de las aplicaciones de forceps corresponden a distocias en el estrecho inferior, ya sea por demora en el desprendimiento o por sufrimiento fetal, que se hace presente en este último periodo del parto. En cuanto al estrecho medio, por lo general las aplicaciones de forceps, obedecen a una falta de rotación o demora en el descenso, y con menor frecuencia al sufrimiento fetal. Aqui el forceps muchas veces se aplica en previsión de que por la demora en la terminación espontánea del parto, éste debe terminarse artificiaimente, con el fin de prevenir el sufrimiento del feto y el desfallecimiento de la madre. Por último, en cuanto se refiere al forceps alto, su empleo. como quedó expuesto, está formalmente contraindicado por algunos obstetras, mientras que otros lo aceptan como uj medio útil de' hacer un parto por las vías genitales sin recurrir a la cesárea, cuando sin existir imposibilidad, absoluta para el encajamiento, éste no se realiza por alguna causa distócica, que pugede ser ven- 
cida por el forceps. En el caso particular de los forceps altos aplicados en la Clínica Restrepo, su empleo obedeció a "distocia de encajamiento" de causa secundaria, debida en un caso a pubis muy alto y promontorio saliente que desviando la cabeza del eje del canal del parto impedían su encajamiento; en otro, la distocia estaba dada por presentación en variedad posterior muy mal flejada con anteversión del útero y contracciones de poca intensidad, y el tercero se debió a retracción uterina; en los tres casos el encajamiento no se efectuaba espontáneamente, en vista de lo cual se resolvió emplear el forceps, con lo cuai se consiguió de una manera fácil la terminación del parto, con resultados muy satisfactorios, obteniéndose niños vivos que salieror de la clínica en magnificas condiciones.

En cuanto a la causa que haya obligado al obstetra a intervenir, podemos considerar varios grupos: aquellas que derivan de una indicación materna, las que provienen del feto, y causas mixtas, que dependen tanto de la madre como del teto. Así, por ejemplo, encontramos indicaciones puramente maternas, en los tres casos de forceps por cardiopatía de la madre, en los cuales, no obstante no existir distocia alguna, el forceps fue indicado con el exclusivo fin de evitar a la madre un esfuerzo violento en el último período del parto, esfuerzo que podía llegar a ser altamente perjudicial, por cuanto, aun cuando existía una cardiopatía perfectamente compensada, era en extremo peligroso someter ese corazón a una prueba demasiado violenta, que llegara a producir la descompensación conduciendo a la paciente a un estado muçho más delicado y peligroso. Las modernas concepciones sobre cardiopatía y embarazo, hacen posible ia coexistencia dé estos dos estados, manteniendo el corazón vigilado durante el pre-natal; sosteniéndolo para prevenir la descompensación, y terminando el período de expulsión mediante la ayuda de un forceps, con el mismo fin de prevenir la descompensación.

Como causas de orden fetal encontramos aquí la procidencia del cordón y el sufrimiento fetal, que se hace presente por la expulsión de meconio y la alteración de los ruidos cardíacos fetales. Nadie discute la necesidad imperiosa de terminar un parto de la manera más rápida en presencia de una procidencia del cordón, que pone en inminente peligro la vida del feto; y en un caso tal la aplicación del forceps se justifica plenamente. En el sufrimiento del feto comprobado, aun cuando algunas de las causas de dicho sufrimiento pueden ser maternas, la indicación del 
forceps es fetal, por cuanto aqui lo que se trata es de intervenis para salvaguardiar la vida al feto, y ningún obstetra vacila en intervenir de esta manera, cuando al frente del sufrimiento fetal patente, no puede terminar el parto rápidamente de otro modo.

En la estadistica de que nos ocupamos encontramos dos casos de procidencia del cordón, y veinticinco de sufrimiento fetal, en algunos de los cuales se comprobó que éste era debido a una o más circulares del cordón. Los demás casos en que e! sufrimiento no se debió a circular alguna, obedecían, bien a un trabajo prolongado generalmente con bolsa rota desde algún tiempo atrás, con fenómenos de compresión cerebral, o a un trabajo demasiado intenso, con anoxia placentaria causada por las mismas contracciones. La anoxia y la compresión se traducen por expulsión de meconio y alteración de los ruidos fetales, debidos a acciones quimicas y mecánicas sobre los centros encefálicos, ya que siendo el centro cardíaco un centro endocraneano, su compresión determina cambios en el ritmo del corazón fetal.

En las historias que comentamos encontramos 27 casos de forceps en los cuales se da como causa la falta de rotación y demora en el descenso de la presentación. Sucede con frecuencia que durante el trabajo de parto éste se prolonga más de lo normal, porque la presentación no rota ni desciende, lo cual sucede más frecuentemente en las variedades de posición posterior. La razón estriba en que en las variedades anteriores, el occipital con su superficie conveja, viene a relacionarse con el arco del estrecho superior en su parte antero lateral, mientras que la frente de superficie más irregular y mayor, se acomoda perfectamente al seno sacro-ilíaco, de tal suerte que la fuerza de expulsión de la contracción uterina y la contrapresión debida a la resistencia pélvica, obran sobre los brazos de palanca desiguales cue forma el diámetro occípito-frontal con punto fijo en la articulación atloido-occipital, haciendo que la cabeza se fleje al mismo tiempo que se hace solidaria del tronco en sus movimientos, do manera que al obedecer éste a las leyes del plano lombo-ilíaco y rotar para poder descender, arrastra a la cabeza en su movimiento de rotación. Mas cuando las condiciones son diferentes y ei occipital se pone en variedad posterior, la frente viene a corresponder al arco anterior del estrecho con deficiente adaptación que iesulta en un movimiento de extensión de la cabeza, que por esta razón viene a presentar un diámetro mayor para el encajamiento, el que no se hace imposible, pero sí más dificil. Ahora bien: al deflejarse dependiente de éste en su movimiento de rotación: el que puede 
efectuarse hacia adelante o hacia atrás, o quedarse indiferente en su primitiva posición y es ésta la razón para que la rotación se dificulte y con ella el descenso, pues la resistenciz que oponen el periné y los estrechos medio e inferior, no es vencida fácilmente por una cabeza que está mal flejada. Es en estos casos en que el periodo de expulsión se prolonga y la rotación no se efectúa, cuando el médico se ve obligado a hacer una aplicación de forceps para vencer la dificultad y evitar al feto un riesgo de muerte, o las secuelas que puede dejar un trabajo largo y laborioso si es que ya no se ha presentado el sufrimiento fetal. En fin, el forceps profiláctico no es más que la consecuencia de io anterior o de la resistencia desproporcionada del periné al paso de la presentación.

Capitulo aparte corresponde al forceps alto, el cual se ha empleado para conseguir el encajamiento de una cabeza que no lo ha logrado, por distocia secundaria, independiente de la desproporción céfalo-pélvica, en la cual algunas veces se ha podido evitar una casárea mediante una aplicación alta de forceps, llenando los requisitos que tan delicada intervención impone; de donde podemos sacar la conclusión de que es factible el forceps alto con éxito, sin que esto quiera decir que toda distocia de encajamiento obedece a la conducta del forceps, pues en riuchos casos se fracasa y en otros, cuando se insiste erróneamente, el resultado es un feto muerto. En apoyo de lo que venimos exponiendo sobre la incidencia del forceps en relación con la causa productora de la distocia, encontramos los datos estadísticos que comentamos, según los cuales, sobre el total de los casos corresponden diez $\mathrm{y}$, siete a variedades de posición anterior y casi todas ligadas a sufrimiento fetal, en la mayoría por circulares del cordón; en cambio hay treinta y seis, es decir, algo más del doble, en variedades posteriores, que dan en la mayoría corno causa del forceps, la falta de rotación y él trabajo prolongado; lo que viene a demostrar que es mucho más frecuente la distocia en las variedades posteriores, o lo que es lo mismo, que éstas son más distócicas que las variedades anteriores. En cuanto a las transversas que encontramos, en muchas de ellas bien pudo tratarse primitivamente de una variedad posterior que se detuvo en la mitad de su rotáción; por lo demás, tanto las transversas como las posteriores son susceptibles más que ninguna otra, al furceps por sufrimiento fetal, por cua zto al prolongarse demasiado el trabajo comienza a hacerse presente el traumatismo obstétrico. 
La paridad es otro factor importante en lo que se refiere a la incidencia del forceps en la Clínica Restrepo, y creo que en cualquier otro medio hospitalario. Naturalmente, de acuerdo con las causas de distocia, éstas son mucho más frecuented en las mujeres primíparas que en las que han tenido más de un parto a término o prematuro por las vías genitales, ya que aquellas en quienes sus embarazos han terminado por abortos de corta edad o por cesáreas, sus vías genitales para el objeto del parto vienen a comportarse prácticamente como de primíparas.

En los casos que comentamos encontramos una proporción muy superior de primíparas que de multíparas, ya que de los 61 casos, corresponden 41 , o sea un $67.20 \%$ a primigrávidas, en contraste con 20 casos, o sea $32.80 \%$ de multigestantes, incluidas entre éstas tres casos, cuyos primeros embarazos terminaron en los primeros meses por abortos, y dos en los que termivaron por cesárea, lo que viene a hacer de ellas prácticamente casos de primíparas; al mismo tiempo que sobre 36 casos en variedad posterior, 28 corresponden a primíparas, o lo que es lo mismo, un $77.8 \%$, contra 8 multíparas, o sea $22.2 \%$; lo cual está demostrando que la necesidad del forceps para variedades posteriores es mucho más alta en las primíparas que, por lo demás, son mujeres en quienes el trabajo del parto es mucho más laborioso

Asimismo influye sobre el trabajo del parto $k$ edad de la parturienta, y especialmente si se trata de primigrávidas, ya que en ellas, mientras más avanzada sea la edad a que sobreviene el primer embarazo, más expuestas están a la distocia y con seguridad más cercanas a un parto intervenido. No es que para la mujer mayor de 28 o 30 años sea imposible tener su primer parto naturalmente; pero sí es más difícil que a una edad menos avanzada, ya que en la mujer añosa se conjugan varias causas de distocia, como son la mala disposición de la fibra uterina para las buenas contracciones y la mayor dureza y menor elasticidad de las partes blandas, que influyen poderosamente en el mecanismo del parto; inclusive el factor psicológico de incapacidad y miedo que invade a la primigestante añosa, en la que puede desarrollarse un verdadero complejo de inferioridad, ya que muchas de ellas saben que son pocas las oportunidades que les quedan para concebir.

Factor de suma importancia en el mecanismo del parto lo constituye la pelvis materna, puesto que es el camino obligado por donde debe transitar el feto en el acto del narimiento; $y$ es lógico admitir que de la constitución y buena disposición de la 
pelvis depende en gran parte el éxito materno, y especialmente fetal. Pero no siempre una buena pelvimetria externa es índice absoluto de pelvis competente, pues existen déficits pélvicos coexistentes con diámetros externos muy buenos, y que no se pueden apreciar sino por la radio-pelvimetría o por lin exploración directa de la pelvis mediante el examen genital. Sin embargo, la medida de los diámetros de la pelvis nos da un índice muy aproximado de la capacidad del basinete, y de ella nos valemos en la mayoría de los casos para apreciar la posibilidad de un parto espontáneo.

En este trabajo hemos tomado solamente el diámetro conjugado externo o de Baudelocque como índice, encontrando los siguientes datos: Baudelocque de 20 o más 'centímetros, 31; de 19 a 20 centímetros, 20; de 18 a 19, 7, y de 17 a 18 centímetros, 3; es decir, que hubo un total de diez casos, o sea un $164 \%$ en los que el diámetro antero posterior del estrecho superior por la pelvimetría externa, estaba muy por debajo de lo normal; sin embargo, el parto fue posible con buen éxito fetal. La radio-pelvimetria se empleó en 9 casos, comprobando por ell $\chi$ dimensiones pequeñas en casi todos los diámetros pélvicos.

Resultados maternos.-En general los resultados de las aplicaciones de forceps con relación a la madre, fueron buenos en todas, sin que se presentara morbimortalidad en ninguna. Como lesiones consecutivas, se presentaron desgarros perineales en 8 casos; en 35 casos se hizo episiotomia, y en 18 no se hizo ni episiotomia ni desgarro. Tanto las episiotomias como ios desgarros, prendieron por primera intención y en buena forma. En cuanto al cuello del útero, no se presentó lesión de ninguna gravedad.

Resultados fitales.-De los 61 fetos, 57 nacieron a término y cuatro prematuros; 1 io que da un $93.4 \%$ para los partos a término, y 6. 6\% para los partos prematuros. De estos niños. 55 respiraron espontáneamente; 5 tuvieron respiración retardada y en ellos hubo necesidad de recurrir a la respiración artificial, y un caso no respiró. En este último se trataba de una paciente que había tenido una ruptura prematura de las membranas, viniendo como consecuencia una fuerte infección amniótica y un trabajo prolongado, "por malas contracciones uterinas. Cuando se extrajo el feto éste estaba muerto; no fue un forceps difícil, iaborioso ni traumatizante, hasta el extremo de matar el feto en la extracción y se realizó una buena toma; de suerte que la muerte se consideró causada por la infección amniótica y por el trauma- 
tismo obstétrico del trabajo prolongado, ya que cuardo se intervino con forceps fue porque se encontraron modificaciones intensas en los ruidos fetales, lo que denotaba el sufrimiento existente.

Muertes neo-natales se presentaron tres; en un caso se trató de muerte de trece horas después del nacimiento por atelectasia pulmonar. Fue el resultado de un embarazo que evolucionó con hidramnios agudo, y en el que se encontraron 4.500 c. c. de lí. quido amniótico; el feto desde el primer momento respiró mal y se cianosó, por lo cual hubo necesidad de colocarlo en incubadora con oxígeno, y prestarle los cuidados necesarins; pero siempre murió, debido a la atelectasia, como se comprobó por autopsia.

El segundo caso de muerte neo-natal se produjo a los cuatro días, por hemorragia endocreneana. Se trató de un embarazo en primigestante, que evolucionó con nefritis crónica y toxemia preeclámptica. Se presentó un parto prematuro, con trabajo de 27 horas. Se aplicó un forceps profiláctico en desprencímiento, sin ningún traumatismo, por lo cual se atribuyó la muerte al traumatismo obstétrico por trabajo prolongado, en un leto prematuro, cuya madre tenía el antecedente de toxemia grave.

Por último, el tercer caso de muerte se produjo nueve horas después del nacimiento. Se trató de un caso de placenta inferior lateral, con hemorragia abundante, en que el feto aspiró bastante cantidad de sangre, produciéndose como consecuencia una afección respiratoria que no se determinó, pero notándose sí que la muerte se debió a un déficit respiratorio, ya que habıa franca disnea con obstáculo a la inspiración y con marcado tiraje.

En total se produjeron 3 muertes neo-natales, lo que da un porcentaje de $4.9 \%$ pero de ninguna de estas muertes puede inculparse directamente al forceps, ya que los hechos demuestran las muertes por otros motivos, pudiéndose pensar que en estos casos la muerte del niño se habría producido fatalmente sin la aplicación del forceps. 